\title{
COMPORTAMENTO BIOMECÂNICO DA PÁLPEBRA RECONSTRUÍDA
}

\author{
BIOMECHANIC BEHAVIOUR OF REPAIRED EYELID
}

\author{
Suzana Matayoshi ${ }^{1}$; Beatriz Yae Hanaoka²; Junko Osaka ${ }^{3}$; \\ Erasmo M. Castro de Tolosa ${ }^{4}$; Nelson Fontana Margarido, TCBC-SP ${ }^{5}$
}

\begin{abstract}
RESUMO: Objetivo: Determinar a contribuição da sutura da borda, tarso e pele na resistência da pálpebra à ruptura pósreconstrução. Métodos: estudo prospectivo, mascarado em coelhos, que foram submetidos à ressecção de um fragmento de espessura total da pálpebra. Os animais foram distribuidos em cinco grupos: G1 sutura da pálpebra em três planos; G2 sutura palpebral sem inclusão do tarso; G3 sutura palpebral sem inclusão da pele; G4 sutura sem inclusão da borda palpebral; G5 sutura da pálpebra igual ao Grupo 1. Os animais do Grupo1 ao 4 foram sacrificados no $7^{\circ}$.dia e os do Grupo 5 no $2^{\circ}$. dia. Os espécimes palpebrais foram submetidos ao teste de rompimento com tensiômetro. Resultados: Foram avaliadas 89 pálpebras provenientes de 85 animais no estudo biomecânico. O grupo no qual a borda palpebral não foi suturada (Grupo 4), houve diminuição estatisticamente significativa de todos os parâmetros analisados: tensão máxima, alongamento e trabalho, em comparação com os demais grupos. Conclusão: A supressão da sutura da palpebral influencia negativamente na resistência tênsil da cicatriz. Havendo a reconstrução da borda palpebral a supressão da sutura tarsal não interfere na biomecânica palpebral. (Rev. Col. Bras. Cir. 2007; 34(1): 31-34).
\end{abstract}

Descritores: Pálpebras; Biomecânica; Reconstrução; Pálpebras/lesões.

\section{INTRODUÇÃO}

Embora o conhecimento das propriedades biomecânicas das pálpebras tenha aplicações importantes nas cirurgias de reconstrução palpebral, existem poucas informações sobre o assunto na literatura médica.

A técnica básica de reconstrução palpebral é representada pela aproximação direta e sutura borda a borda. Classicamente recomenda-se o reparo da margem palpebral em três etapas: o fechamento da margem palpebral com três suturas principais (transição cutâneo-mucosa, linha cinzenta e linha dos cílios), a sutura do tarso anterior e a sutura cutânea ${ }^{1}$. Situações clínicas relativamente comuns no atendimento de pacientes são aquelas representadas por suturas inadequadas de pálpebra: suturas incompletas de margem e tarso.

Em estudo prévio, verificou-se que o modelo experimental de reconstrução e cicatrização palpebral em coelhos reproduz as alterações encontradas em trauma palpebral humano $^{2}$.

Fizemos este estudo com o objetivo de determinar a contribuição da sutura da borda, tarso e pele na resistência da pálpebra à ruptura pós-reconstrução.

\section{MATERIAL E MÉTODOS}

Estudo prospectivo, experimental, mascarado conduzido em coelhos, no qual foi realizada cirurgia palpebral com ressecção de um fragmento com espessura total no cen- tro da pálpebra inferior (Figura 1) e reconstrução borda a borda (Figura 2).

Os animais foram distribuídos em cinco grupos: G1sutura clássica da pálpebra (borda, tarso e pele); G2 - sutura da borda e da pele;sem tarso; G3 - sutura da borda e do tarso;sem pele; G4 - Sutura do tarso e da pele, sem borda palpebral. G5 - Sutura do tarso, pele e borda palpebral.

Os animais dos Grupos 1 ao 4 foram submetidos a exame ocular externo e sacrifício no $7^{\circ}$ dia pós-operatório, os animais do Grupo 5 no $2^{\circ}$ dia pós-operatório. As pálpebras inferiores eram seccionadas, obtendo-se espécimes de $20 \mathrm{~mm}$

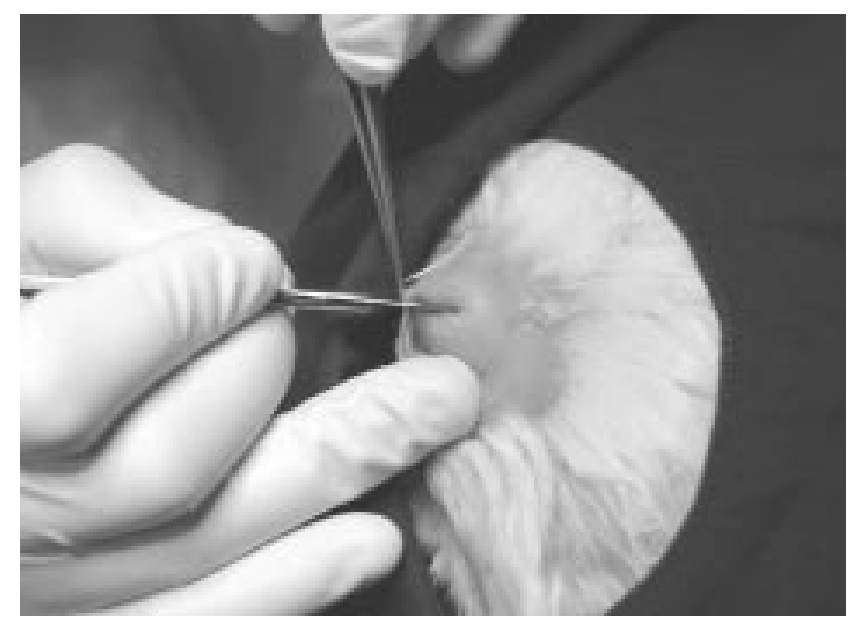

Figura 1 - Ressecção de um fragmento com espessura total no centro da pálpebra inferior.

\footnotetext{
1. Assistente-doutora da Clínica Oftalmológica do HCFMUSP.

2. Médica Residente da Disciplina de Técnica Cirúrgica e Cirurgia Experimental da FMUSP.

3. Biomédica da Disciplina de Técnica Cirúrgica da FMUSP.

4. Ex-Professor da Disciplina de Técnica Cirúrgica e Cirurgia Experimental da FMUSP.

5. Professor Associado e Professor Responsável pela Disciplina de Técnica Cirúrgica e Cirurgia Experimental da FMUSP.

Recebido em 29/06/06

Aceito para publicação em 01/08/06
}

Conflito de interesses: nenhum

Fonte de financiamento: nenhuma

Trabalho realizado na Disciplina de Técnica Cirúrgica da FMUSP. 


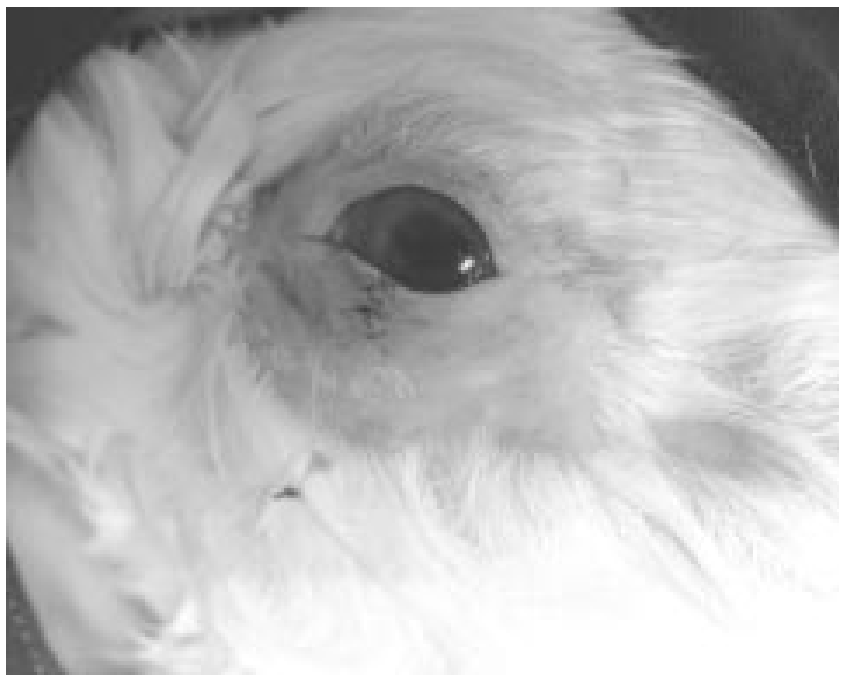

Figura 2 - Pálpebra reconstruída com suturas em posição.

x $5 \mathrm{~mm}$ e imediatamente encaminhadas para avaliação biomecânica (Figura 3). Esta avaliação consistia em submeter os espécimes palpebrais ao teste de rompimento em tensiômetro, acoplado a um computador. As pálpebras eram presas ao tensiômetro a partir de suas duas extremidades laterais. Desta forma, as bordas da cicatriz cirúrgica eram tracionadas lateralmente, em sentidos opostos.

O tensiômetro utilizado (Strain gage mod.P.A. 06364350 Kratos) era composto por uma célula de carga e numa bomba de infusão adaptada (Figura 4). A célula de carga consiste numa base de alumínio com um conjunto de resistências elétricas que formam uma ponte de Wheatstone. Quando esta célula é submetida a tração ou a compressão, ocorrem deformações na sua estrutura. Isto gera variações de potencial que podem ser calibrados para corresponderem a um determinado valor de peso, em gramas. A bomba de infusão, por sua vez, é responsável pela tração do corpo de prova a uma velocidade constante.

Durante cada ensaio, os registros transmitidos para o computador pelo tensiômetro eram plotados em um gráfico. O eixo das abcissas representava o tempo de tração decorrido e o eixo das ordenadas, a tensão aplicada no corpo de prova. Desta forma foram extraídas informações relativas ao compor-

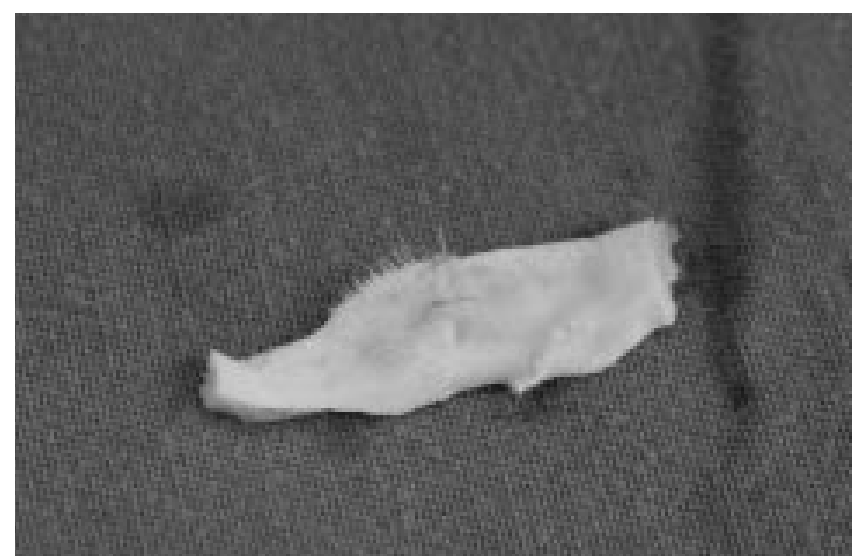

Figura 3 - Espécime palpebral ressecado para a avaliação biomecânica. tamento biomecânico das pálpebras de coelhos reconstruídas. As seguintes características biomecânicas foram avaliadas:

1 - Força máxima (em "dina"): igual ao maior valor de força suportada pelo corpo de prova durante todo o ensaio realizado.

2 - Alongamento na força máxima (em $\mathrm{cm})$ : igual à distância percorrida pelo braço da bomba de infusão entre o início do ensaio até o instante em que se atingiu a força máxima suportada pelo corpo de prova.

3 - Trabalho da força máxima (em "erg"): Corresponde ao produto da força máxima pelo alongamento no instante do registro da força máxima, dividido por dois.

\section{RESULTADOS}

No estudo biomecânico, foram avaliadas 89 pálpebras provenientes de 85 animais, distribuídos em cinco grupos. O número de animais em cada grupo foi variável: Grupo 1 - 25 animais, Grupo 2 -19 animais, Grupo 3 -16 animais, Grupo 4 - 11 e grupo $5-14$ animais.

Houve deiscência de sutura em duas pálpebras (uma no Grupo 1 e outra no Grupo 2). Além disso, cinco pálpebras (quatro do Grupo 1 e uma do Grupo 2) apresentaram irregularidades de margem palpebral, com desnivelamento ântero-posterior das bordas da cicatriz.

A Tabela 1 mostra os resultados de três parâmetros que foram analisados neste estudo: tensão máxima suportada pelo tecido antes da ruptura; o alongamento tecidual medido antes da ruptura e o trabalho necessário para produzir a ruptura tecidual.

A Tabela 2 mostra os resultados da análise estatística comparativa (teste de Student) entre os cinco grupos. Podese observar, portanto, que os dados correspondentes ao Grupo 4 diferiram significativamente em relação aos demais grupos em relação a todos os parâmetros analisados. A tensão máxima, alongamento e trabalho necessários para provocar ao ruptura palpebral foram menores do que as necessárias para outros grupos. $\mathrm{O}$ alongamento nos animais do Grupo 5 foi maior que no Grupo 1, mas os outros parâmetros não tiveram diferença estatisticamente significativa.

A aplicação do teste T de Student nos dados é mostrada na tabela abaixo:

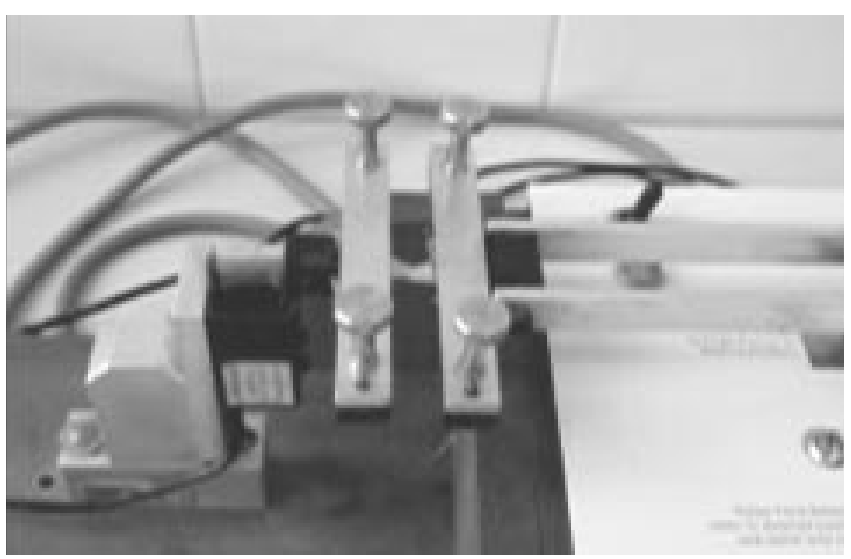

Figura 4 - Tensiômetro mostrando a pálpebra em posição para o ensaio biomecânico. 
Tabela 1 - Resultados da avaliação da tensão palpebral. Os valores apresentados correspondem à média e desvio-padrão dos diversos grupos.

\begin{tabular}{lccc}
\hline Grupo & $\begin{array}{c}\text { Tensão máxima } \\
\left(\mathbf{1 0}^{\mathbf{7}} \text { dinas } / \mathbf{c m}^{\mathbf{2}} \pm \text { i.c. }\right)\end{array}$ & $\begin{array}{c}\text { Alongamento } \\
(\mathbf{c m}) \pm \mathbf{i . c} .\end{array}$ & $\begin{array}{c}\text { Trabalho } \\
\left.\mathbf{( 1 0}^{\mathbf{5}} \mathbf{~ e r g}\right)\end{array}$ \\
\hline 1 & $0,81 \pm 0,14$ & $8,74 \pm 1,33$ & $4,62 \pm 1,85$ \\
2 & $0,80 \pm 0,16$ & $9,51 \pm 1,21$ & $4,73 \pm 1,40$ \\
3 & $0,95 \pm 0,19$ & $10,33 \pm 1,50$ & $6,42 \pm 2,02$ \\
4 & $\mathbf{0 , 4 9} \pm \mathbf{0 , 1 1}$ & $\mathbf{6 , 3 0} \pm \mathbf{1 , 7 4}$ & $\mathbf{1 , 5 9} \pm \mathbf{0 , 7 6}$ \\
5 & $0,77 \pm 0,16$ & $10,67 \pm 1,06$ & $4,37 \pm 1,08$ \\
\hline
\end{tabular}

Obs. Os dados significativos foram assinalados em negrito.

\section{DISCUSSÃO}

As lesões palpebrais são freqüentes em serviços de urgência. $\mathrm{O}$ conhecimento das propriedades biomecânicas da pálpebra tem aplicações importantes nas operações de reconstrução palpebral. Entretanto não encontramos na literatura consultada estudos sobre a contribuição da sutura da borda, tarso e pele.

A técnica básica de reconstrução palpebral é a sutura por planos ( borda, tarso e pele) utilizada em casos de trauma palpebral e na reconstrução palpebral em defeitos de borda e pós ressecção de neoplasias ${ }^{4}$. No presente estudo simularam-se algumas situações clínicas, que são relativamente encontradas no atendimento de uma laceração palpebral: sutura completa; sutura da margem e pele, mas não do tarso. $\mathrm{O}$ terceiro grupo foi constituído por animais onde se realizou a sutura do tarso e margem mas não da pele, com o objetivo de avaliar o efeito da sutura da pele na tensão final pós-reconstrução. O Grupo 4 não foi submetido à sutura da borda palpebral para determinar a importância da sutura da borda palpebral na resistência tênsil da pálpebra pós-reconstrução O Grupo 5 foi avaliado após 48 horas pois procurou-se estabelecer qual seria a influência do tempo no processo de cicatrização.

Estudo anterior mostrou que a pálpebra de coelho é semelhante à humana para fins de investigação biomecânica, embora ressaltem-se algumas diferenças como.o tamanho reduzido do tarso, o músculo orbicular pouco desenvolvido e uma pálpebra comparativamente mais fina que a humana ${ }^{2}$.

A análise dos três primeiros grupos não mostrou diferença, ou seja, deixar de suturar o tarso (Grupo 2) ou a pele (Grupo 3) não ocasionou a alterações biomecânicas significativas. $\mathrm{O}$ resultado surpreende pois, esperar-se-ia que o fato de não suturar o tarso, propiciaria uma cicatrização irregular e portanto uma pálpebra menos resistente. Como já foi dito anteriormente, o fato do coelho apresentar um tarso mais fino assim como uma maior proximidade entre as duas lamelas palpebrais, pode eventualmente explicar uma cicatrização normal mesmo sem a sutura tarsal.

Já em relação ao Grupo 4 no qual a borda palpebral não foi suturada, observou-se uma diminuição estatisticamente significativa de todos os parâmetros analisados: tensão máxima, alongamento e trabalho, em comparação com os demais grupos.

Isto pode significar que em termos de estrutura palpebral, a integridade da borda é o parâmetro mais relevante na cicatrização, em conformidade com dados da literatura. Clinicamente, Mustardé demonstrou a importância da borda para a manutenção da forma e da fisiologia da abertura e fechamento palpebral. $\mathrm{O}$ efeito de laminação, ou seja, a rigidez palpebral seria conferida pelo efeito da adesão das lamelas posterior e anterior, pela borda palpebral, que teria uma estrutura reforçada em comparação ao resto da pálpebra ${ }^{5}$.

A resistência de uma cicatriz operatória, medida pela tensão máxima, é um parâmetro bastante empregado na avaliação do processo de cicatrização. Sabe-se que durante os três primeiros dias de síntese de uma ferida, a resistência tênsil da lesão (definida pela resistência à tração por unidade de área de tecidos) depende quase que exclusivamente da capacidade de sustentação das suas bordas. Após o terceiro dia, iniciava-se ocorrer a síntese do colágeno pelos fibroblastos concomitantemente com o aumento da resistência tênsil da ferida ${ }^{6}$ e nas primeiras duas semanas de cicatrização, a resistência da cicatriz é diretamente proporcional à quantidade de colágeno depositada ${ }^{7}$.

Tabela 2 - Análise estatística (teste T de Student).

\begin{tabular}{|c|c|c|c|}
\hline Comparação & $\begin{array}{c}\text { Tensão máxima } \\
\left(\mathbf{1 0}^{7} \text { dinas } / \mathrm{cm}^{2} \pm \text { i.c. }\right)\end{array}$ & $\begin{array}{l}\text { Alongamento } \\
(\mathrm{cm}) \pm \text { i.c. }\end{array}$ & $\begin{array}{l}\text { Trabalho } \\
\left(10^{5} \text { erg) }\right.\end{array}$ \\
\hline $1 \times 2$ & $\mathrm{t}=0,099$ & $\mathrm{t}=0,1883$ & $\mathrm{t}=0,097$ \\
\hline $1 \times 3$ & $t=1,261$ & $t=1,643$ & $\mathrm{t}=1,352$ \\
\hline $1 \times 4$ & $\mathrm{t}=3,048 *$ & $\mathrm{t}=2,270 *$ & $\mathrm{t}=2,294 *$ \\
\hline $1 \times 5$ & $\mathrm{t}=0,392$ & $\mathrm{t}=\mathbf{2 , 2 0 3} *$ & $\mathrm{t}=0,217$ \\
\hline $2 \times 3$ & $\mathrm{t}=1,293$ & $\mathrm{t}=1,105$ & $\mathrm{t}=1,704$ \\
\hline $2 \times 4$ & $\mathrm{t}=\mathbf{2 , 8 4 4} *$ & $\mathrm{t}=3,262 *$ & $\mathrm{t}=3,247^{*}$ \\
\hline $2 \times 5$ & $\mathrm{t}=0,283$ & $\mathrm{t}=1,454$ & $\mathrm{t}=0,404$ \\
\hline $3 \times 4$ & $\mathrm{t}=3,966^{*}$ & $\mathrm{t}=3,770 *$ & $\mathrm{t}=4,076 *$ \\
\hline $3 \times 5$ & $\mathrm{t}=1,475$ & $t=0,684$ & $\mathrm{t}=1,905$ \\
\hline $4 \times 5$ & $\mathrm{t}=\mathbf{3 , 5 0 0} *$ & $\mathrm{t}=4,949 *$ & $\mathbf{t}=4,094 *$ \\
\hline
\end{tabular}

(*) estatisticamente significativo. 
É interessante notar que no presente estudo, mesmo em apenas dois dias, não houve diferenças biomecânicas da pálpebra de coelho comparadas com o grupo de sete dias de cicatrização. A resposta ao trauma ocular em coelhos é, geralmente, mais agressiva e mais rápida do que em seres humanos ${ }^{8}$, talvez as alterações cicatriciais também se comportem dessa forma .

$\mathrm{O}$ alongamento palpebral do Grupo 5 foi maior que $o$ Grupo 1, mas os outros parâmetros não foram estatisticamen- te significativos, portanto este resultado não foi considerado para a análise.

Nas condições do presente estudo, pode-se afirmar que a supressão da sutura da borda palpebral influenciou negativamente na resistência tênsil da cicatriz. Verificou-se também que desde que a borda seja reconstruída, a supressão da sutura tarsal não interfere na biomecânica palpebral.

\begin{abstract}
Objective: To determine the importance of sutures of the eyelid border, tarsus and skin and their resistance to rupture after reconstruction. Methods: A prospective study was carried out in rabbits submitted to total thickness resection of the eyelid. The animals were divided into 5 groups: G1 (eyelid suture in 3 layers); G2 (eyelid suture excluding tarsus); G3 (eyelid suture excluding skin), G4 (suture excluding the eyelid border), G5 (eyelid suture as in G1). The animals from group 1 to 4 were killed on the $7^{\text {th }}$ day, while those from group 5 were killed on the $2^{\text {nd }}$ day. The palpebral specimens underwent rupture test with a tensiometer. Results: 89 eyelids from 85 animals were analyzed. The group in which the border was not sutured (group 4) showed a significant reduction in all studied parameters: maximum tension, stretching and work, as compared with the other groups. Conclusion: The lack of suture in the eyelid border had a negative impact in scar tensile resistance. Additionally, we showed that if the eyelid border is repaired, the avoidance of tarsal suture does not interfere with the eyelid biomechanics.
\end{abstract}

Key words: Eyelids; Biomechanics; Reconstruction; Eyelids/injuries.

\section{REFERÊNCIAS}

1. Lemke, BN e Della Rocca, RC Eyelid margin and tarsus: Anatomy and surgery IN Lemke, BN e Della Rocca . Surgery of the eyelids and orbit - an anatomical approach. London. Prentice-Hall.1990. p.211-2

2. Hanaoka, B.Y.; Ikeda, M. H.; Osaka, J; Tolosa, E.C.M., Matayoshi, S; Margarido, N.F. Estudo experimental do comportamento biomecânico da pálpebra reconstruída. Apresentado no XXI Congresso Médico Universitário (COMU). São Paulo, Brasil. 2002

3. Soares,L.A Processo de cicatrização das feridas na parede abdominal: influência da sutura do plano peritoneal com fio de categute: estudo experimental [tese]. São Paulo, Faculdade de Medicina da Universidade de São Paulo, 1991

4. Matayoshi.S; Santanna, AEB; Bessa, H, Ferreira Filho, N. Moura, EM. Anatomia Cirúrgica da Órbita In Soares, E; Moura, EM; Gonçalves,JO. Ed.. Cirurgia Plástica Ocular. São Paulo: Roca, $1997:$ p.7

5. Mustardé, JC. Repair and reconstruction in the orbital region. 3rd.Edition Edinburgh: Churchill Livinsgstone; 1991
6. Neto, João Elias Calache. Efeitos de administração do diclofenaco sódico a ratos submetidos a suturas cólicas [Tese]. Faculdade de Medicina da Universidade de São Paulo,1990

7. Ehrlich, H.P., et al; Vanadate ingestion increases the gain in wound breaking strength and leads to better organized collagen fibers in rats during healing. Plastic and Reconstructive Surgery, vol. 107, no. 2, p. 471-477, 2001

8. Miller, M.H.; Grierson, I.; Unger, W.I.; Hitchings, R.A. Wound healing in an animal model of glaucoma fistulizing surgery in the rabbit. Ophthalmic Surg., v. 20, p. 350-7, 1989.

Como citar este artigo:

Matayoshi S, Hanaoka BY, Osaka J, Tolosa EMC, Margarido NF. Comportamento biomecânico da pálpebra reconstruída. Rev Col Bras Cir. [periódico na Internet] 2007;34(1). Disponível em URL: http:// www.scielo.br/rcbc

Endereço para correspondência:

Nelson Fontana Margarido

Rua Itapeva, 378 - cj. 22

Bela Vista

01332-000 - São Paulo - SP 